\title{
Algorithms for Solving Misalignment Issues in Penalized PET/CT Reconstruction Using Anatomical Priors
}

\author{
Yu-Jung Tsai, Student Member, IEEE, Alexandre Bousse, Sangtae Ahn, Member, IEEE, Charles W. \\ Stearns, Fellow, IEEE, Simon Arridge, Brian F. Hutton, Senior Member, IEEE and Kris Thielemans, Senior \\ Member, IEEE
}

\begin{abstract}
In dual-modality systems, using anatomical priors has been shown to improve image quality and quantification in emission tomography. However, alignment between the functional and anatomical images is crucial. In this study, we propose two algorithms for solving misalignment issues. Both approaches are based on a recently published joint motion estimation and image reconstruction method. The first approach deforms the anatomical image to align it with the functional one while the second approach deforms both images to align them with the measured data. Our current implementation uses alternates between image reconstruction and alignment estimation. To evaluate the potential of these approaches, we have chosen Parallel Level Sets (PLS) as a representative anatomical penalty since it has shown promising results in literature, incorporating a spatially-variant penalty strength to achieve uniform local contrast and fast convergence rate. The performance evaluation was achieved by using simulated non-TOF data generated with an XCAT phantom in the thorax region. We used the attenuation image in the anatomical prior. The results demonstrated that both methods are able to estimate the misalignment and deform the anatomical image accordingly when a proper workflow for the alternating optimization is applied. However, the performance of the first approach depends highly on the workflow of the alternating process. In contrast, the second approach shows the ability to converge to the correct alignment faster than the first approach does, independent of the workflow. Our results indicate that it is possible to align functional and anatomical information, enabling the use of anatomical priors in practice.
\end{abstract}

Manuscript received December 18, 2018. This work was supported by GE Healthcare and by the National Institute for Health Research, University College London Hospitals Biomedical Research Centre.

Yu-Jung Tsai is with the Institute of Nuclear Medicine, University College London, London, UK (e-mail: yu-jung.tsai.14@ucl.ac.uk).

Alexandre Bousse is with the Institute of Nuclear Medicine, University College London, London, UK. He is also with the LaTIM, INSERM, UMR 1101, Université de Bretagne Occidentale, Brest, France (e-mail: a.bousse@ucl.ac.uk).

Sangtae Ahn is with GE Global Research, Niskayuna, NY 12309 USA (e-mail: ahns@ge.com)

Charles W. Stearns is with MICT Engineering, GE Healthcare, Waukesha, WI 53188 USA (e-mail: Charles.Stearns@med.ge.com).

Simon Arridge is with the Department of Computer Science, University College London, London, UK (e-mail: S.Arridge@cs.ucl.ac.uk).

Brian F. Hutton is with the Institute of Nuclear Medicine, University College London, London, UK. He is also with the Centre for Medical Radiation Physics, University of Wollongong, Wollongong, Australia (e-mail: b.hutton@ucl.ac.uk).

Kris Thielemans is with the Institute of Nuclear Medicine, University College London, London NW1 2BU, UK (e-mail: k.thielemans@ucl.ac.uk).

\section{INTRODUCTION}

Penalized positron emission tomography (PET) image reconstruction using penalties derived from anatomical images, such as computed tomography (CT) or magnetic resonance (MR) images, has been shown to be effective in improving object delineation and reducing quantitative error in many studies [1]. However, to utilize the structural information without incurring artifacts, a good alignment between the anatomical and the functional images is essential. This is challenging in practice in the thorax because scanners obtain these images separately or sequentially. Even with a multimodality scanner that performs simultaneous functional and anatomical image acquisition such as in PET/MR, alignment can be difficult to achieve due to the different time scales of the PET and MR scans. According to the results in [2], wrong spatial information from the misaligned anatomical image can lead to ghost artifacts and low quantitative accuracy. To be able to use anatomical penalties in clinical practice, we propose two approaches that estimate the misalignment and deform the anatomical image accordingly during the reconstruction.

\section{METHOD}

\section{A. Objective function without misalignment}

Penalized image reconstruction optimizes a function $\Phi$ consisting of the likelihood $L$ and the penalty function $R$, with a constant parameter $\beta$ which controls the strength of the penalty. As Parallel Level Sets (PLS) has shown promising results in the literature [3], it was chosen as a representative anatomical penalty function in this study. In addition to using $\beta$ to regulate the global weight of the penalty term, a spatiallyvariant penalization map $\kappa$ was also incorporated into the penalty function for achieving uniform local contrast across the field-of-view (FOV). Benefits of applying $\kappa$ with PLS were demonstrated in our previous work [4]. Given the PET image $\boldsymbol{x}$, the anatomical image $\boldsymbol{v}$ and the measured data $\boldsymbol{y}$, the objective function is:

$$
\begin{gathered}
\Phi(\boldsymbol{x})=-L(\boldsymbol{x}, \boldsymbol{\mu})+\beta R(\boldsymbol{x} \mid \boldsymbol{v}), \\
R(\boldsymbol{x} \mid \boldsymbol{v})=\sum_{j} \kappa_{j}^{2} \sqrt{\alpha^{2}+\left\|[\nabla \boldsymbol{x}]_{j}\right\|_{2}^{2}-\left\langle[\nabla \boldsymbol{x}]_{j},[\boldsymbol{\xi}]_{j}\right\rangle^{2}} \\
\boldsymbol{\kappa}=\sqrt{D\left[\boldsymbol{A}^{\top} D\left[\frac{\boldsymbol{y}}{\left(\boldsymbol{A} \boldsymbol{x}^{0}+\boldsymbol{n}\right)^{2}}\right] \boldsymbol{A 1}\right]},[\boldsymbol{\xi}]_{j}:=\frac{[\nabla \boldsymbol{v}]_{j}}{\sqrt{\left\|[\nabla \boldsymbol{v}]_{j}\right\|_{2}^{2}+\eta^{2}}}
\end{gathered}
$$


where $\boldsymbol{x}^{0}$ is the initial image, $\boldsymbol{n}$ is the expected background and $\boldsymbol{A}$ is the system matrix where every element $A_{i j}$ denotes the probability that an emission from voxel $j$ is detected by bin $i$. $\boldsymbol{A}$ depends on the estimated attenuation $\mu$. The strength of the edge preserving property of the penalty function is modulated by the set of parameters $[\alpha, \eta]$. The notation $D$ and $\nabla$ represent diagonal and gradient operators, respectively.

\section{B. Objective function with misalignment}

In this study, we propose two approaches that account for the misalignment between the functional and anatomical images by incorporating a warp matrix $\boldsymbol{W}$ into the penalized objective function $\Phi$ in (1). Both approaches are based on a joint motion estimation and image reconstruction method proposed recently for dealing with the mismatch between the attenuation map and the PET image in respiratory gated PET/CT [5]. The main difference between them is that the first approach $\Phi_{1}$ warps the anatomical image to align it with the functional image, while the second approach $\Phi_{2}$ warps both images to align them with the measured data. The deformation of the images is described by a cubic B-spline function with a collection of coefficients $\boldsymbol{\theta}$ in this study. Assume the attenuation map $\boldsymbol{\mu}$ is used for providing anatomical information as well, the approaches can be described as follows:

$$
\begin{aligned}
\Phi_{1}(\boldsymbol{x}, \boldsymbol{W}) & =-L(\boldsymbol{x}, \boldsymbol{W} \boldsymbol{\mu})+\beta R(\boldsymbol{x} \mid \boldsymbol{W} \boldsymbol{\mu})+\gamma Q(\boldsymbol{\theta}) \\
\Phi_{2}(\boldsymbol{x}, \boldsymbol{W}) & =-L(\boldsymbol{W} \boldsymbol{x}, \boldsymbol{W} \boldsymbol{\mu})+\beta R(\boldsymbol{x} \mid \boldsymbol{\mu})+\gamma Q(\boldsymbol{\theta})+\delta B(\boldsymbol{W} \boldsymbol{x}), \\
B & =\sum_{j} \sum_{m} \min \left(0, \sum_{l} w_{l}\left(z_{m}\right) x_{j l}\right)^{2}
\end{aligned}
$$

where $Q(\boldsymbol{\theta})$ represents a quadratic penalty on the B-spline coefficients for reducing the influence of noise and $\gamma$ is a constant that controls its strength. We used a non-negativity constraint for the pixel values. In the first approach, this is done using constrained optimization. For the second approach, we are optimizing the B-spline coefficients not pixel values. Therefore, we introduced a barrier function $B$ to penalize negative values [6] $\boldsymbol{x}$ after warping. Given $\boldsymbol{z}=\left\{z_{m} \mid m=1, \ldots, M\right\}$ a vector containing a finite number of uniformly spaced locations in each interknot interval, the function computes the spline value (i.e., the image value) at each location and penalizes the square of any negatives. Although the warped $\boldsymbol{\mu}$ might have negative values as well, we have ignored this as small negative values in $\boldsymbol{\mu}$ would become attenuation factors very close to one. The strength of $B$ is determined by the parameter $\delta$ and $M=4$ in this study. The notation $w_{l}$ represents the $\ell$ th basis weight of the B-spine function. The optimization of both approaches is achieved by an alternating process between the deformation estimation and the penalized image reconstruction.

\section{Data}

To evaluate the approaches, we produced 2 XCAT phantoms representing different respiratory phases and the corresponding $\boldsymbol{\mu}$ maps. Both phantoms were a $128 \times 128 \times 47$ matrix with voxel size of $3.906 \mathrm{~mm}$. We generated data corresponding to that from a GE Discovery STE in 3D non-TOF acquisition mode. The misalignment estimation for non-TOF data is much harder than that for TOF data.

\section{Reconstruction}

The optimization of both approaches is an alternating process that includes a misalignment estimation subroutine and an image reconstruction subroutine. We applied limitedmemory Broyden-Fletcher-Goldfarb-Shanno (L-BFGS) for unconstrained optimization (misalignment estimation in both approaches and image reconstruction in the second approach) and L-BFGS-B [7] for the positivity constrained image reconstruction in approach 1 . To improve the convergence rate of the penalized image reconstruction, a preconditioner proposed in our previous study was also incorporated [8]. The workflow is defined by the number of inner iterations for these two subroutines and the number of outer iterations that controls the repetition of the alternating process. The whole optimization was initialized by one full iteration of OSEM with 14 subsets and the $\boldsymbol{\mu}$ map at end expiration was used as the initial input of the misalignment estimation. Every time the misalignment estimation is done, a new initial image for L-BFGS-B-PC is recomputed using OSEM with 14 subsets, taking into account the current estimated misalignment. The $\kappa$ image, which is the spatially variant penalty strength, is recomputed at every outer iteration as well. In a preliminary investigation, we explored the influence of the parameters of the algorithms. We found that reasonable results can be obtained for both approaches by using up to 100 outer iterations with 1 and 10 inner iterations for the misalignment and image reconstruction subroutines, respectively. The set of parameters that determine the strength of each penalty function was $(\beta, \gamma)=\left(10^{-1}, 10^{-4}\right)$ for $\Phi_{1}$ and $(\beta, \gamma, \delta)=\left(10^{-1}, 10^{-4}, 10^{-1}\right)$ for $\Phi_{2}$. To illustrate the performance dependence of each approach on different workflows, we also show the reconstructed images with 15 inner iterations for the misalignment estimation, keeping all other workflow and parameter settings unchanged.

\section{E. Evaluation}

Both approaches should be able to find a warped attenuation map similar to that used for data generation (i.e., $\boldsymbol{\mu}$ at end inspiration). In this study, we use the difference image between the warped and target $\boldsymbol{\mu}$ maps to evaluate the performance of the misalignment subroutine. The reconstructed functional images at 100 outer iterations are also provided.

\section{RESULTS}

Fig. 1 shows the difference images and the corresponding reconstructed activity images for both approaches at 100 outer iterations. The applied workflow was 1 inner iteration for the misalignment estimation and 10 inner iterations for the penalized image reconstruction. As demonstrated in the difference images, both approaches are able to estimate the misalignment and warp the input attenuation map accordingly. The reconstructed activity images are visually similar to the target one, but they are somewhat smoother due to the implied interpolation of image warping using a B-spline function. The corresponding results for the workflow with 15 inner iterations for the misalignment estimation are shown in Fig. 2. As observed in the difference image, the first approach still suffers from the misalignment issue at 100 outer iterations leading 

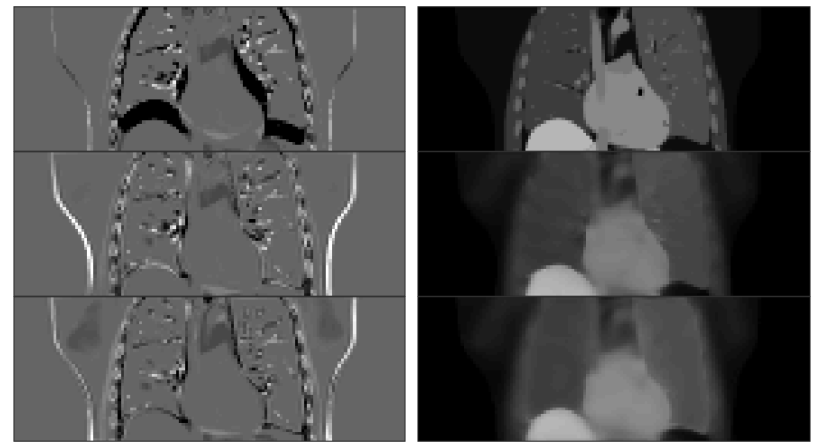

Fig. 1: The difference image between the target and input $\boldsymbol{\mu}$ maps and the true activity image (first row). The difference images between the target and $\boldsymbol{W} \boldsymbol{\mu}$ maps and the corresponding activity images at 100 outer iterations are also provided (second row for approach 1 and bottom row for approach 2). The applied workflow was 1 and 10 inner iterations for the misalignment estimation and image reconstruction.
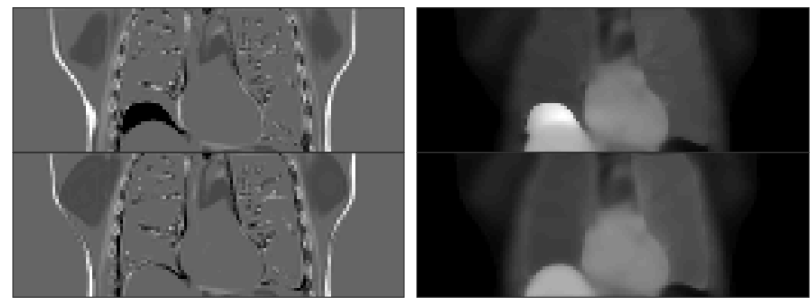

Fig. 2: The difference images between the target and $\boldsymbol{W} \boldsymbol{\mu}$ maps and the corresponding activity images for both approaches (top row for approach 1 and bottom row for approach 2) at 100 outer iterations for the workflow with 15 inner iterations for the misalignment estimation.

to a banana artifact in the reconstructed activity image (top row). In contrast, the second approach is insensitive to the change of workflow as both its difference and the reconstructed functional images (bottom row) look similar to those in Fig. 1.

\section{DISCUSSION}

We have demonstrated that the proposed approaches are able to estimate the misalignment and warp the anatomical image accordingly. When a proper workflow is applied, satisfactory results with no apparent artifacts can be obtained in 100 outer iterations for both approaches. By comparing the results to those reconstructed without using PLS, the use of the additional anatomical information showed the ability to improve the convergence rate of the misalignment estimation for approach 2 but slow it down for approach 1 (not shown). As illustrated in Fig. 2, the performance of approach 1 depends highly on the workflow while approach 2 is able to converge to the correct alignment, independent of the workflow. Although not shown, we also observed that the second approach requires less outer iterations to converge to a reasonable result than the first approach. Future work includes further evaluation of performance dependence on other factors, such as the initial condition and strength of each penalty.

\section{REFERENCES}

[1] K. Vunckx, A. Atre, K. Baete, A. Reilhac, C. Deroose, K. V. Laere, and J. Nuyts, "Evaluation of Three MRI-Based Anatomical Priors for
Quantitative PET Brain Imaging," IEEE Trans. Med. Imag., vol. 31, no. 3, pp. 599-612, 2012.

[2] M. J. Ehrhardt and S. R. Arridge, "Vector-Valued Image Processing by Parallel Level Sets," IEEE Trans. Med. Processing, vol. 23, no. 1, pp. $9-18,2014$.

[3] M. J. Ehrhardt, P. Markiewicz, M. Liljeroth, A. Barnes, V. Kolehmainen, J. S. Duncan, L. Pizarro, D. Atkinson, B. F. Hutton, S. Ourselin, K. Thielemans, and S. R. Arridge, "PET Reconstruction With an Anatomical MRI Prior Using Parallel Level Sets," IEEE Trans. Medical Imag., vol. 35, no. 9, pp. 2189-99, 2016

[4] Y.-J. Tsai, G. Schramm, J. Nuyts, S. Ahn, C. W. Stearns, A. Bousse, S. Arridge, and K. Thielemans, "Spatially-variant Strength for Anatomical Priors in PET Reconstruction," IEEE Nucl. Sci. Symp. and Med. Imag. Conf. Rec., 2017.

[5] A. Bousse, O. Bertolli, D. Atkinson, S. Arridge, S. Ourselin, B. F. Hutton, and K. Thielemans, "Maximum-Likelihood Joint Image Reconstruction/Motion Estimation in Attenuation-Corrected Respiratory Gated PET/CT Using a Single Attenuation Map," IEEE Trans. Medical Imag., vol. 35, no. 1, pp. 217-28, 2016.

[6] T. E. Nichols, J. Qi, E. Asma, and R. M. Leahy, "Spatiotemporal reconstruction of list-mode PET data," IEEE Trans. Med. Imag., vol. 21, no. 4, pp. 396-404, 2002.

[7] R. H. Byrd, P. Lu, J. Nocedal, and C. Zhu, "A Limited Memory Algorithm for Bound Constrained Optimization," SIAM J. Sci. Comput., vol. 16, no. 5, pp. 1190-208, 1995.

[8] Y.-J. Tsai, A. Bousse, M. J. Ehrhardt, C. W. Stearns, S. Ahn, B. F. Hutton, S. Arridge, and T. K., "Fast quasi-Newton algorithms for penalized reconstruction in emission tomography and further improvements via preconditioning," IEEE Trans. Med. Imag., vol. 37, no. 4, pp. 1000-10, 2018. 